\title{
Hypercanes: A possible link in global extinction scenarios
}

\author{
Kerry A. Emanuel, ${ }^{1}$ Kevin Speer, ${ }^{2}$ Richard Rotunno, ${ }^{3}$ Ramesh Srivastava, ${ }^{4}$ and \\ Mario Molina ${ }^{1}$
}

\begin{abstract}
Bolide impacts and large-scale volcanic eruptions have been proposed as possible causes of the massive extinction of life that has occurred episodically in Earth's history. In spite of the catastrophic disruption of the local environment that accompanies bolide impacts and volcanic eruptions, it has been difficult to explain why these events sometimes lead to global extinction of species. We propose that, in some cases, the missing link may be provided by hypercanes, runaway hurricanes that are capable of injecting massive amounts of water and aerosols into the middle and upper stratosphere, where they may have profound effects on atmospheric chemistry and radiative transfer. Hypercanes are theorized to occur when the sea surface temperature exceeds a critical threshold, which may occur when sea water is locally heated by bolide impact, shallow-sea volcanism, or possibly, by overturning of superheated brine pools formed by underwater volcanic activity. Simulations using a convection-resolving nonhydrostatic, axisymmetric numerical model show that hypercanes can indeed develop when the sea surface temperature is high, and that they inject large amounts of mass into the stratosphere.
\end{abstract}

\section{Introduction}

Evolution of life has been punctuated by events during which large numbers of species have become extinct. Largest among the known events was the Late Permian extinction, about 245 m. y. ago, during which about $96 \%$ of all species disappeared. The most recent major extinction occurred at the end of the Cretaceous, between 64 and $66 \mathrm{~m}$. y. ago, and was marked by the disappearance of about three fourths of all species that existed at that time. Two hypotheses have emerged to explain the end-Cretaceous extinction. Alvarez et al. [1980] proposed that the impact of a large asteroid led to the massive extinction, while Officer and Drake [1983] and others argue that the extinction was related to massive volcanic eruptions that resulted in the formation of the Deccan Traps. It has also been suggested that these two geophysical events are related [Alt et al., 1988].

Considerable evidence has accumulated in recent years in support of the idea that the Earth was struck by one or more bolides at the end of the Cretaceous. Foremost among such evidence is the finding by Alvarez et al. [1980] that iridium and other platinum group metals known to be more concentrated in extraterrestrial bodies than on Earth, are enriched in a thin clay layer at the Cretaceous-Tertiary boundary worldwide. Other evidence includes the finding that this clay layer contains extraterrestrial amino acids [Zahnle and Grinspoon, 1990], soot attributed to global wildfires [Wolbach et al., 1985], and an Osmium isotopic ratio with a distinctly extraterrestrial signal

\footnotetext{
'Center for Meteorology and Physical Oceanography, Massachusetts Institute of Technology, Cambridge.

${ }^{2}$ Institut Français de Recherche pour l'Exploitation de la Mer, Brest, France.

${ }^{3}$ National Center for Atmospheric Research, Boulder, Colorado.

${ }^{4}$ Department of Geophysical Sciences, University of Chicago, Chicago, Illınois.

Copyright 1995 by the American Geophysical Union.

Paper number 95JD01368.

0148-0227/95/95JD-01368\$05.00
}

[Luck and Turekian, 1983]. Many Cretaceous-Tertiary (K/T) boundary sequences also contain abundant shocked mineral grains that suggest an impact, although it has also been proposed that this may result from explosive volcanism. Recent attention has focused on the Chixslub feature on the northern Yucatan coast as a possible impact crater, though several other craters can also be dated to the $\mathrm{K} / \mathrm{T}$ transition, suggesting that multiple impacts may have occurred in a short time. There is also evidence in $\mathrm{K} / \mathrm{T}$ sediments of a large tsunami event in the Caribbean region.

Although the evidence for the impact of at least one large bolide at the end of the Cretaceous is mounting, there remains considerable controversy about just how such an event might lead to massive extinction of life. The original Alvarez et al. [1980] paper suggested that the hypothesized asteroid impact would have thrown up a large quantity of dust into the stratosphere, where it would have reduced substantially the incoming solar radiation, leading to a dramatic decrease of photosynthesis and concomitant disruption of the food chain.

Other means by which the impact might have led to mass extinctions include: (1) dramatic, direct warming of the atmosphere and/or ocean by the impact $[H s \dot{u}, 1980]$; (2) global cooling owing to soot produced by global wildfires [Wolbach et al., 1985]; (3) chemical contamination of the atmosphere and/or upper ocean [Hsü, 1980]; (4) global warming by large increases in atmospheric $\mathrm{CO}_{2}$ owing to the impact on carbonate terrain [O'Keefe and Ahrens, 1989]; and (5) global acid rain owing to large increases in atmospheric sulfur and nitrates [Prinn and Fegley, 1987]. As an alternative to the bolide impact hypothesis, large-scale volcanism has also been cited as a possible cause of mass extinctions. Of particular interest are flood basalt fissure eruptions, which are capable of producing individual lava flows with volumes greater than $100 \mathrm{~km}^{3}$ at very high eruption rates. It is speculated that such large eruptions would inject massive amounts of debris into the stratosphere where it might lead to reduced sunlight and depletion of ozone, and that immense quantities of acid rain would reduce the alkalinity and $p \mathrm{H}$ of the upper ocean.

The mass extinctions were evidently selective. The weight of 
evidence suggests that the most dramatic extinctions occurred among the planktonic foraminifers [D'Hondt and Keller, 1991] and calcareous nannoplankton [Thierstein, 1981], and a major mollusk group disappeared entirely [Ward, 1988]. But diatoms, dinoflagellates, and other dwellers of the upper ocean suffered only modest reductions. The planktonic flora and fauna appeared to undergo a rather sudden decline. There were also large reductions in land animals, particularly among the larger species (e.g., dinosaurs), but considerable controversy surrounds the question of the timing of such reductions, and many smaller animals survived the K/T transition.

Many, although not all, of the proposed mechanisms by which bolide impacts and/or large-scale volcanism lead to mass extinctions rely on massive injections of material into the stratosphere, where it may remain for many years. By contrast, injections of large quantities of aerosols into the troposphere should have only transient and local effects, since excess material is washed out of the troposphere on a timescale of weeks. The original Alvarez et al. [1980] thesis contends that large amounts of material from the colliding bolide and from the crust would find its way to the stratosphere, perhaps by being mechanically injected through the "hole" punched in the atmosphere by the incoming bolide. To our knowledge, this conjecture has never been subjected to rigorous tests.

Another conjecture holds that significant amounts of mass entered the stratosphere through hot plumes generated directly through the heat of impact or the combustion of surface material. The volcanic hypothesis also relies heavily on thermal plumes to loft material into the stratosphere, where it can have long-term effects. But the altitude to which thermal plumes from areally limited surface sources ascend in the stratosphere increases only as the $\frac{1}{4}$ power of the surface heat flux [Morton et al., 1956]. It is thus highly unlikely that global wildfires could affect the stratosphere in a significant way, even if there were many of them, since the surface heat flux per unit area is vastly insufficient to achieve stratospheric altitudes. Moreover, even the very largest of historical eruptions (e.g., Krakatoa in 1883) managed to achieve only limited penetration into the stratosphere, such that the material settled out in a matter of a few years (see, for example, Budyko et al., [1988]). Moreover, the settling time of aerosols in the stratosphere may have an upper bound, owing to the fact that the very smallest particles have diameters comparable to or smaller than the mean free path at those altitudes [Reitmeijer, 1993]. It is not obvious that either large-scale volcanism or bolide impacts could loft enough material into the stratosphere for long enough periods to have global effects of a magnitude necessary to explain mass extinctions.

Our present purpose is to suggest that in at least some cases of oceanic massive volcanism or bolide impact, hypothetical atmospheric storms known as hypercanes may have played an essential role in injecting material into the stratosphere. Hypercanes are extraordinarily intense hurricanes whose energy production is so large that it cannot be balanced by surface dissipation, resulting in storms that are so intense that internal dissipation becomes important. These storms are hypothesized to occur when the degree of air-sea thermodynamic equilibrium exceeds a theoretically defined threshold value. Their circulations would penetrate to high altitudes in the stratosphere, where they could deposit large quantities of mass, including water vapor, condensed water, and volcanic ash.

After reviewing the theory of hypercanes, we present the first numerical simulations of these storms using a fully non-

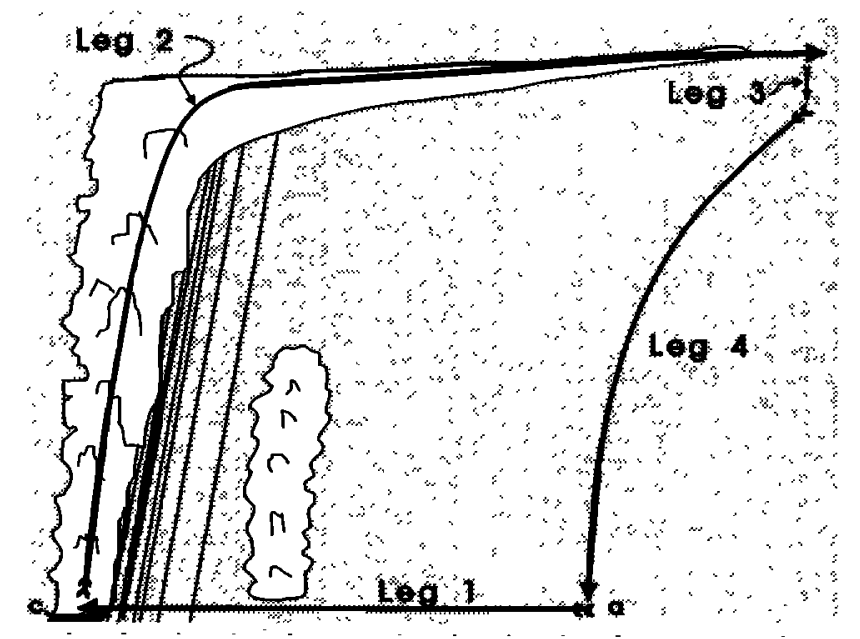

Figure 1. The Carnot cycle of a mature tropical cyclone. Air spirals inward from the environment a to near the center c, while acquiring entropy from the sea surface. Air then ascends adiabatically and flows out to large radius, where it eventually loses heat by radiation to space along legs 3 and 4 .

hydrostatic model, lending credence to the idea that hypercanes are physically possible. We then discuss plausible scenarios in which hypercanes might have occurred as a result of bolide impact or undersea volcanism. We conclude by speculating about the effects of hypercanes on the stratosphere and how these effects may have helped cause mass extinctions.

\section{Hypercanes}

\subsection{Theory}

The energy cycle of a mature hurricane can be characterized as that of a Carnot heat engine [Emanuel, 1986], as illustrated in Figure 1. In its normal state, the tropical atmosphere is quite far from being in thermodynamic equilibrium with the underlying ocean. This disequilibrium is necessary to support the large rates of heat loss by evaporation needed to balance the incoming solar radiation. (Little heat is lost directly by infrared radiation, owing to the opacity of the moist atmosphere above, leaving evaporation as the principal means of disposing of heat.) The disequilibrium is reflected in the subsaturation of air just above the sea surface; typical relative humidities are about $80 \%$.

As the ambient air spirals into the storm center in a thin boundary layer, the wind speed increases and so too does the rate of evaporation. In the strongest hurricanes, the air near the core comes close to a state of thermodynamic equilibrium with the ocean, reflecting a large heat gain on its inward journey. This heat gain is observed to occur at nearly constant temperature. Having reached the core, the air spirals upward in a ring of convection known as the "eyewall," which surrounds a circular region of nearly cloud-free air known as the "eye." This upward motion represents a nearly adiabatic expansion of the working fluid, which is a mixture of moist air and suspended condensed water droplets. These droplets ultimately combine into particles large enough to fall out of the system as precipitation. The air then flows out at high altitude to large distances from the storm center, after which it loses the heat it gained from the ocean by radiating it to space, or in an open-cycle system, by exporting it to other atmospheric 


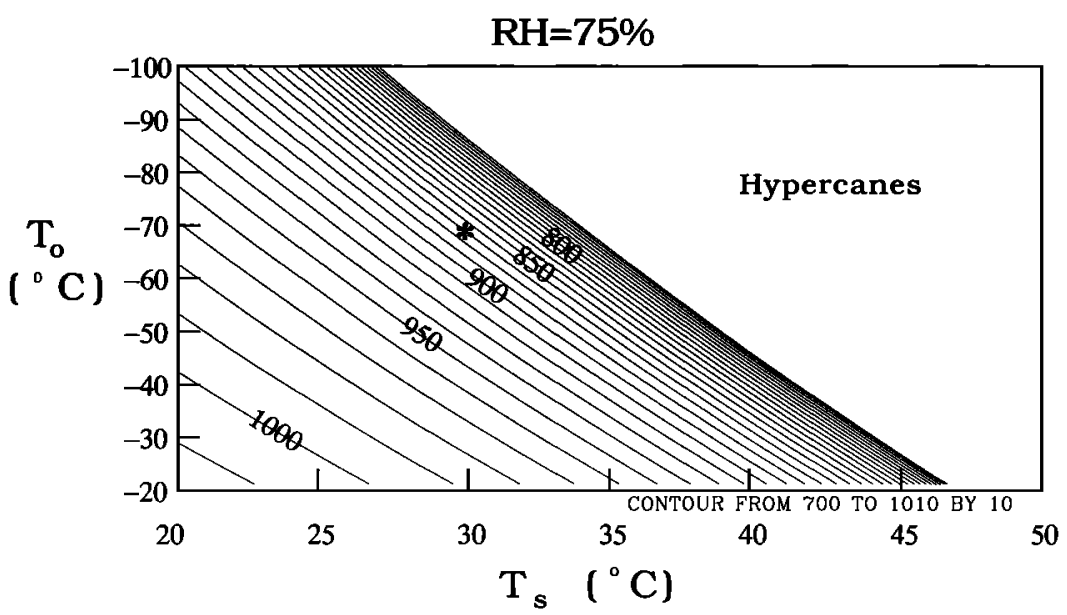

Figure 2. Minimum central pressure (mbar) of tropical cyclones calculated from (1), with ambient surface relative humidity of $75 \%$. The asterisk denotes conditions typical of the tropical Pacific. Normal sea level pressure is 1013 mbar, while the lowest pressure ever recorded in a tropical cyclone is 870 mbar.

circulation systems. Finally, the air descends back to the surface, losing more heat by electromagnetic radiation. But since the vertical profile of air temperature in the environment of the storm is nearly that of adiabatic ascent of moist air with suspended water droplets, this additional heat loss is almost exactly that which would have occurred if the condensed water had been retained and was now reevaporating.

If the working fluid is considered to consist of a mixture of dry air, water vapor, and condensed water, the energy cycle may be closely approximated by isothermal expansion of air spiraling inward along the ocean surface, followed by adiabatic expansion of air ascending in the eyewall, followed by isothermal compression of air losing heat at the storm top, and followed finally by adiabatic compression to the starting point. The thermodynamic efficiency of the engine is proportional to the temperature drop from the sea surface to the lower stratosphere, where the outflow occurs. For typical conditions this temperature drop is of the order of $100 \mathrm{~K}$, while the inflow temperature is roughly $300 \mathrm{~K}$, giving an efficiency of about one third.

The total increase in enthalpy of the inflowing air is limited by the degree of air-sea thermodynamic disequilibrium of the ambient air: When this air reaches thermodynamic equilibrium, no further heat input is possible. (Such an equilibrium is characterized by equal sea and air temperatures and air relative humidity of $100 \%$.) This provides an upper bound on the energy input to the system.

In the mature hurricane the energy input balances dissipation. Most of this dissipation occurs in the thin, turbulent boundary layer above the sea surface and is proportional to the total pressure drop from the ambient environment to the storm center. A smaller amount of energy is lost at large radii in the outflow, where the low angular momentum of the outflow (which has been previously lost to the sea in the inflow) must be restored to environmental values by turbulence. A yet much smaller, but poorly known, amount of energy is lost in turbulence in the interior of the system.

Neglecting this last sink, the energy balance may be written as an equation for the central surface pressure of the storm, which is a direct measure of its intensity. Given the aforementioned bound on the heat input, there is a corresponding bound on the surface pressure drop from the ambient environment to the center. Emanuel [1988] developed an implicit equation for this drop, in the form

$$
\ln (x)=-(A / x)+B
$$

where $x$ is the ratio of the central value of the partial pressure of dry air to its ambient value, and $A$ and $B$ are coefficients that depend on the ambient degree of air-sea thermodynamic disequilibrium, the thermodynamic efficiency, and the storm radius, which determines how much energy is dissipated in the outflow.

Solutions to (1) are graphed in Figure 2 as a function of the sea surface temperature and the outflow temperature, assuming a fixed environmental surface relative humidity of $75 \%$. (The surface relative humidity is observed to vary little with local climate conditions over the ocean; then the sea surface temperature determines the degree of air-sea thermodynamic disequilibrium.) The asterisk indicates conditions typical of the warmest regions of the tropics. Computations of this same bound for climatologically normal conditions (see, for example, Emanuel, [1986]) show that a small fraction of real hurricanes come very close to their energy bound. On the other hand, accurate numerical simulations of hurricanes (see, for example, Rotunno and Emanuel, [1987]) seem to always produce storms at the theoretical limit of intensity. Nonaxisymmetric interactions of real storms with their environments and local reduction of sea surface temperature, owing to turbulent mixing of surface waters with deeper, colder water, limit most, but not all, storms to intensities well below the theoretical limit.

Figure 2 also shows that there are no solutions to (1) for certain values of the coefficients $A$ and $B$. Close analysis of the system shows that in the supercritical regime where no solutions to (1) are possible, the Carnot cycle is unstable, owing to the the component of heat input by isothermal expansion. The lower the central surface pressure, the more heat is added to the inflow by isothermal expansion; this added heat intensifies the vortex, leading to further reduction of the central surface pressure, more heat input by isothermal expansion, and so on. This effect is so strong in the supercritical regime that the additional surface dissipation cannot balance it, and the vortex 
intensifies until internal dissipation becomes sufficient to balance the energy input. The resulting system is what we have called a hypercane.

To date, the only numerical simulation of a hypercane was that of Emanuel [1989], who used a very simple model consisting of only three layers of atmosphere and which made several restricting assumptions about the force balances in the flow. This nevertheless showed that in the hypercane regime of parameter space, the vortex continues to intensify until numerical stability criteria are exceeded, and the integration had to be stopped. Here we present results of integrations in the hypercane regime using a much more sophisticated model.

\subsection{Numerical Simulations}

We performed several integrations of the numerical hurricane model developed by Rotunno and Emanuel [1987]. It is designed to simulate the axisymmetric circulation of hurricanes, including the circulation within convective clouds, though the latter are forced to be axisymmetric rings. This model integrates the fully nonhydrostatic, compressible equations on an axisymmetric domain $1000 \mathrm{~km}$ in radius and $45 \mathrm{~km}$ in altitude, with radial and vertical resolutions of $5 \mathrm{~km}$ and 1.25 $\mathrm{km}$, respectively. The model also integrates conservation equations for heat and water, but condensed water is represented by only one category, the total liquid water mixing ratio. The terminal velocity of the falling liquid water is taken to be $7 \mathrm{~m}$ $\mathrm{s}^{-1}$ if the water content exceeds $1 \mathrm{~g} \mathrm{~kg}^{-1}$ and zero otherwise; this is the crudest way of handling rain while retaining only one category of condensate. One drawback to this approach is that evaporation of falling rain must proceed at a pace sufficient to keep the air saturated; this exaggerates the rate of evaporation. A sponge layer occupying the top $5 \mathrm{~km}$ of the domain serves to partially absorb internal wave activity, which in nature would radiate into the high atmosphere without much back reflection.

All turbulence in the model is represented by an eddy viscosity that depends on the local rate of deformation and the local Richardson number, the latter of which is defined with a dry or moist static stability, depending on whether the air is unsaturated or saturated. Surface fluxes of heat, moisture, and momentum are represented using the classical bulk aerodynamic formulae, but with wind-dependent exchange coefficients (all of which are equal). Radiative cooling is crudely mimicked by a Newtonian relaxation back to the initial condition, with maximum values limited to $2 \mathrm{~K} \mathrm{~d}^{-1}$.

A few approximations to the equations that are considered minor for simulations of ordinary hurricanes may prove to be problematic in the hypercane regime. In the construction of the model equations the pressure and potential temperature are separated into parts representing the background state and deviations from it, the former being functions of altitude alone. The full pressure gradient acceleration, given by $-c_{p} \theta_{v} \nabla \pi$, is replaced in the model by $-c_{p} \overline{\theta_{v}} \nabla \pi$, where $c_{p}$ is the heat capacity at constant pressure, $\theta_{v}$ is the virtual potential temperature, and $\pi$ is the nondimensional pressure perturbation. The overbar indicates the background state value. This approximation neglects the quadratic terms in the pressure and buoyancy perturbations. In normal hurricanes these perturbations are of the order of $10 \%$ of the mean values, so the neglect of the quadratic terms is justified. In hypercanes, however, the perturbations are of the same order as the mean values, and so this approximation breaks down.

Although sound waves are meteorologically unimportant, they are explicitly calculated in the model. The terms in the equations that govern the propagation of sound are integrated on a smaller time step than the other terms. This may be considered a special way of calculating mass continuity that avoids the solution of an elliptic equation at the expense of integrating some of the terms on a smaller time step. In the present case the effective mass continuity equation is the standard anelastic equation, in which the background density, rather than full density, is employed. This can be shown to be a good approximation as long as the Mach number of the flow is small. In the case of hypercanes, however, the Mach number is of order unity, and the anelastic approximation breaks down.

The two approximations discussed above are probably not serious problems before and just after the transition of the model storm to the hypercane regime, in which the central surface pressure is lower than the critical transition value, but they become problematic as the hypercane approaches full intensity. Thus while we feel that the model in its present form shows that the transition to the hypercane is indeed a physical possibility, the characteristics of the mature storm should be viewed as preliminary.

With one exception the initial condition is identical to that used by Rotunno and Emanuel [1987], consisting of a tropical atmosphere and sea surface temperature that has been adjusted so that the atmosphere is effectively neutral to moist convection. This mimics the real state of the atmosphere in which moist convection is in statistical equilibrium with largescale processes, such as radiative cooling of the atmosphere and surface heat fluxes, that act to destabilize the flow to convection. Such a state is nearly but not exactly neutral to convection. It is an example of self-organized criticality. The stratosphere in the initial state is isothermal.

Onto this state we superimpose a bell-shaped axisymmetric sea surface temperature anomaly with a decay scale of $100 \mathrm{~km}$. The maximum sea surface temperature at the center is $50^{\circ} \mathrm{C}$, decaying to the ambient value of $27^{\circ} \mathrm{C}$ at large radii. This anomaly is intended to represent the result of local ocean warming by bolide impact or undersea volcanism; since its scale is assumed to be much smaller than an atmospheric deformation radius, the ambient atmosphere as a whole will not adjust appreciably to the anomaly.

As in the work by Rotunno and Emanuel [1987], an initial vortex with maximum wind speeds of $12 \mathrm{~m} \mathrm{~s}^{-1}$ is used to initiate the finite-amplitude instability that results in tropical cyclones. (Later we shall see that the large temperature gradients generated by the sea surface temperature anomaly are sufficient to initiate a storm from very small amplitude initial conditions.)

Figure 3 shows the evolution of the central surface pressure and maximum azimuthal wind with time. After a short time the initial vortex undergoes rapid intensification, with central pressures lower than 300 mbar at around 25-40 hours. Afterward, the vortex weakens somewhat and approaches a slowly decaying state with very strong winds. Experiments in which the cap on the radiative cooling rate was removed show that the resulting storms reach a nearly steady state. The slow decay in the control experiment at long time is a result of the inability of the system to lose heat at a sufficient rate.

The spatial distributions of various quantities in the mature hypercane at 50 hours into the simulation are shown in Figure 4. These fields have been averaged over a 5-hour period. Surface radial inflow in the boundary layer reaches peak values in excess of $50 \mathrm{~m} \mathrm{~s}^{-1}$, while outflow near the tropopause has a maximum value of $60 \mathrm{~m} \mathrm{~s}^{-1}$. Although most of the outflow 


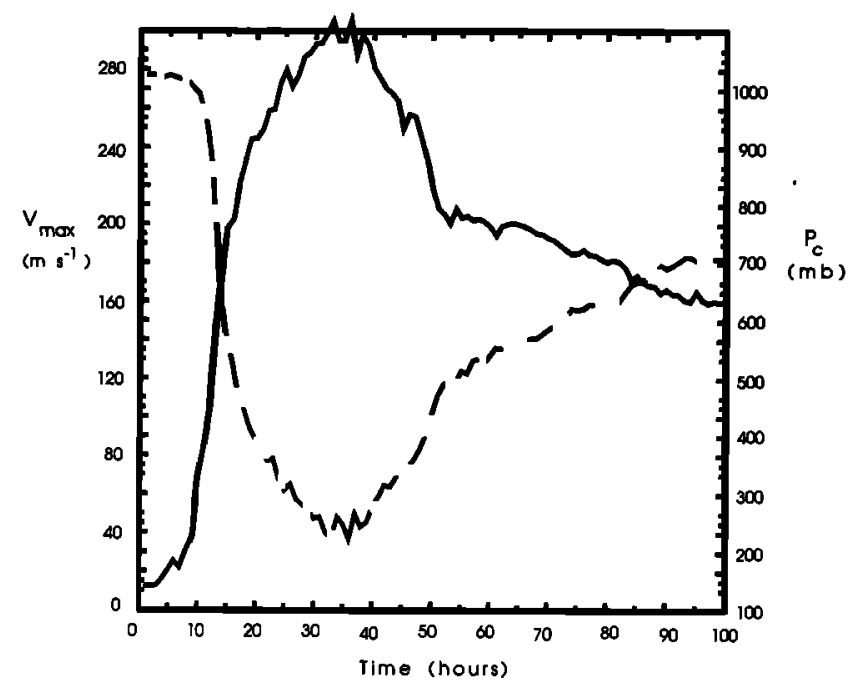

Figure 3. Evolution of the maximum azimuthal velocity (solid line) and minimum pressure (dashed line) in the main experiment.

occurs near the tropopause, some convection in the eyewall reaches altitudes greater than $30 \mathrm{~km}$, and weak outflow can be detected there. The large penetration depths are achieved because of the very high specific entropy contents reached by air spiraling into the core. Figure 4d shows that the equivalent potential temperature (a quantity whose natural logarithm is proportional to the total specific entropy) of air rising in the eyewall has values in excess of $700 \mathrm{~K}$. Such large values result from the nearly isothermal expansion of air flowing into regions of very low pressure in the eye.

The azimuthal wind component is shown in Figure $4 \mathrm{~b}$. The maximum value of $220 \mathrm{~m} \mathrm{~s}^{-1}$ occurs at the surface at a radius of $6 \mathrm{~km}$, and the cyclonic circulation extends up to about $35 \mathrm{~km}$ altitude.

At 50 hours the vertical velocity (Figure 4c) reaches peak values in excess of $35 \mathrm{~m} \mathrm{~s}^{-1}$, though at other times values greater than $70 \mathrm{~m} \mathrm{~s}^{-1}$ occurred. These are comparable to vertical velocities achieved in the most severe middle latitude thunderstorms, and are about an order of magnitude greater than are typical in normal hurricanes. Descent occurs at the innermost grid point (the eye), and weak descent characterizes most of the outer region of the simulated storm.

The water vapor mixing ratio (Figure $4 \mathrm{e}$ ) shows extreme values of over $50 \mathrm{~g} \mathrm{~kg}^{-1}$ in the core, reflecting the low partial pressure of dry air there. Condensed water mixing ratios well over $10 \mathrm{~g} \mathrm{~kg}^{-1}$ extend well up into the stratosphere (Figure 4f).

Several experiments were performed to test the sensitivity of the hypercane evolution to the size and magnitude of the sea surface temperature anomaly; these are summarized in Figure 5. As the geometric size of the sea surface temperature anomaly decreases, the maximum wind speed decreases but is still very substantial when the decay scale is only $25 \mathrm{~km}$. The mature circulation is somewhat steadier when the scale of the sea surface temperature anomaly is small. When the peak value of the ocean temperature is reduced, the maximum wind speed falls but is still substantial when the peak sea surface temperature is $42^{\circ} \mathrm{C}$.

Although the approximations in the model physics render suspect some features of the mature hypercane circulation, these approximations should not be of great importance near the transition from hurricane to hypercane, suggesting that hypercanes are physically possible. The experiments suggest that sea surface temperatures of greater than about $45^{\circ} \mathrm{C}$ occurring on scales greater than about $50 \mathrm{~km}$ may be capable of supporting hypercanes. It remains to be seen whether sea surface temperature anomalies of this size and magnitude are capable of spontaneous ignition of hypercanes, or whether an independent disturbance or "trigger" is necessary, as is certainly the case with ordinary hurricanes. To help answer this question, we ran an experiment identical to the control but beginning with a warm core vortex with maximum winds of only $2 \mathrm{~m} \mathrm{~s}^{-1}$. This initial vortex amplified to the same intensity as the control simulation with only a minor delay, showing that the atmospheric temperature gradients associated with the sea surface temperature anomaly are sufficient for spontaneous generation of storms.

We have assumed that the ambient atmosphere is calm and that it has the thermodynamic characteristics of the present tropical atmosphere. But significant vertical wind shear is known to inhibit or prevent the development of hurricanes, and there is no reason to suspect that this is not the case with hypercanes, whose early evolution is similar to that of hurricanes. At the very least, the presence of mean wind, even if it is does not vary with altitude, will move the storm across the ocean surface. In order for hypercanes to develop, the mean wind would have to be small enough relative to the size of the sea surface temperature anomaly to permit the storm to develop in the time it takes to cross the anomaly. Given a development time of 40 hours, mean winds would have to be less than about $1 \mathrm{~m} \mathrm{~s}^{-1}$ to allow hypercane development over a $100-\mathrm{km}$-scale sea surface temperature anomaly. Such conditions are unusual, but not unknown in the deep tropics. But it is unlikely that hypercanes would develop over localized sea surface temperature anomalies of the kind likely to have been produced by bolide impact or volcanism if the mean winds are much stronger than this. Given weak but nonvanishing tropospheric winds, we conjecture that a sequence of hypercanes would develop, with each member developing rapidly over the sea surface warm anomaly, moving away, and decaying while another forms.

\section{Environmental Effects of Hypercanes}

The most significant characteristic of hypercanes, from the standpoint of environmental impact, is their ability to inject large amounts of mass into the middle stratosphere, where it may remain for many years. If we assume, on the basis of the numerical simulations, that a ring of air between 5 and $35 \mathrm{~km}$ radius is ascending at a rate of $5 \mathrm{~m} \mathrm{~s}^{-1}$ at an altitude of $20 \mathrm{~km}$ (see Figure 4c), then the net mass flux into the middle stratosphere is about $10^{10} \mathrm{~kg} \mathrm{~s}^{-1}$. This is enough to replace the entire mass of the atmosphere between the 100- and 50-mbar pressure surfaces in about 6 months. Two aspects of this mass transport are of particular interest: (1) the flux of water substance, which has the potential of radically altering radiative transfer through the stratosphere and of affecting atmospheric chemistry and (2) the flux of aerosols, which also may alter radiative transfer.

\subsection{Stratospheric Water}

The critical issue here is how much water ascends in the main hypercane updraft without precipitating out of the system. The minimum absolute temperature in the updraft occurs 


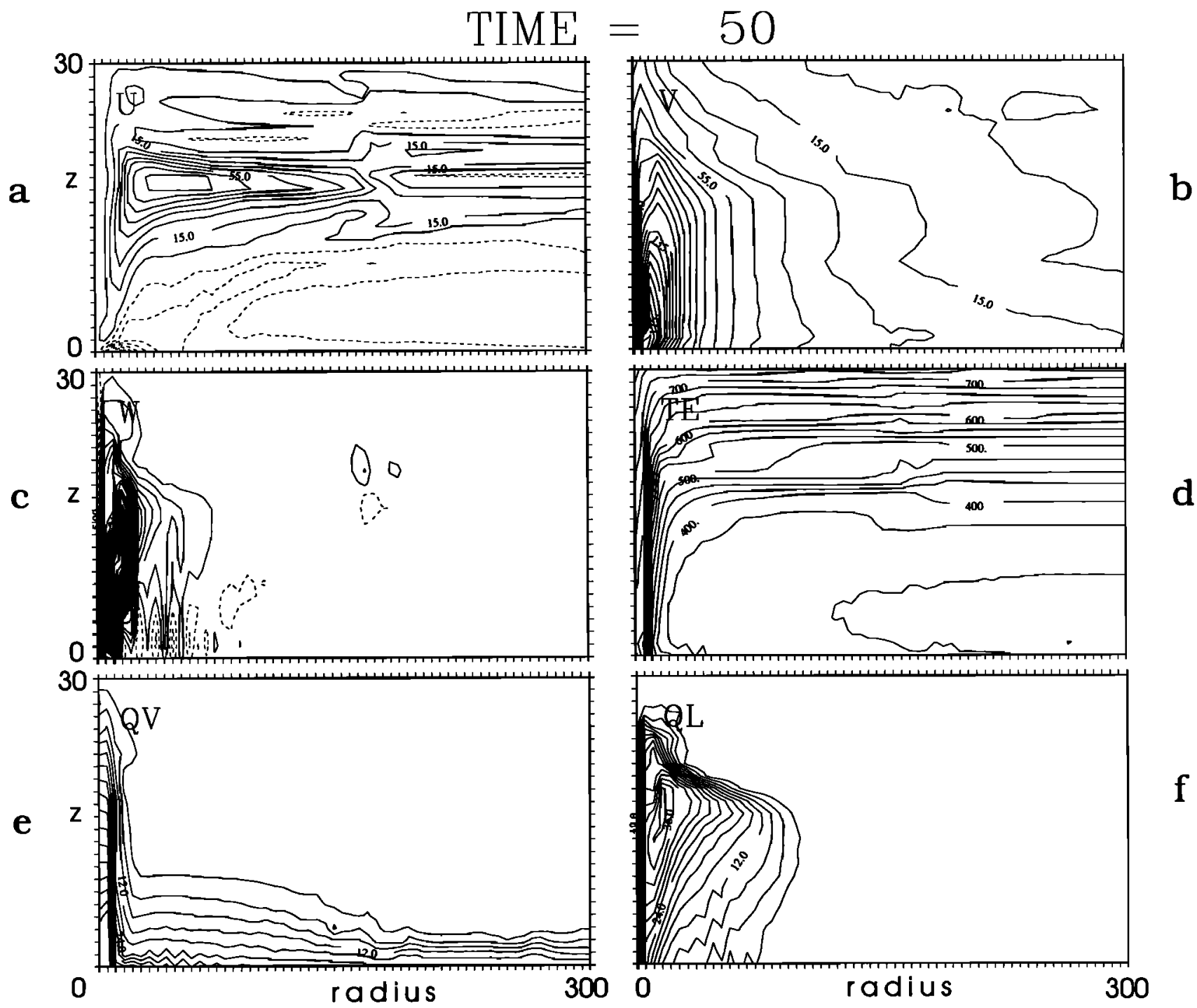

Figure 4. Distributions of variables at 50 hours into the main experiment in a domain extending radially to $300 \mathrm{~km}$ and vertically to $30 \mathrm{~km}$. (a) Radial velocity in meters per second with contour interval of $10 \mathrm{~m} \mathrm{~s}^{-1}$. (b) Azimuthal velocity in meters per second with contour interval of $10 \mathrm{~m} \mathrm{~s}^{-1}$. (c) Vertical velocity with contour interval of $2 \mathrm{~m} \mathrm{~s}^{-1}$. (d) Equivalent potential temperature with contour interval of $25 \mathrm{~K}$. (e) Water vapor mixing ratio in grams per kilogram with contour interval of $3 \mathrm{~g} \mathrm{~kg}^{-1}$. (f) Liquid water mixing ratio in grams per kilogram with contour interval of $3 \mathrm{~g} \mathrm{~kg}^{-1}$.

near the altitude of the outflow; this is about $-70^{\circ} \mathrm{C}$. At the tropopause, where outflow in normal hurricanes is concentrated, this temperature corresponds to a saturation water vapor mixing ratio of about $30 \mathrm{ppm}$, but at $26-\mathrm{km}$ altitude the saturation mixing ratio is closer to $100 \mathrm{ppm}$, owing to the lower total pressure. Thus assuming that the updraft is saturated, somewhat more water vapor is present in the hypercane updraft. One peculiarity of the hypercane environment is that the saturation mixing ratio actually increases with altitude in the nearly isothermal stratosphere; thus any hypercane effluent that begins to sink isothermally through the lower stratosphere under the influence of radiative cooling will experience a decrease of saturation mixing ratio with time. Thus further condensation of water may occur in the descending air.

Of potentially far greater importance is the flux of condensed phase water at high altitudes in the hypercane updraft. At the low temperatures of the outflow region, all of the condensed water would be in the ice phase; a critical issue is how much of the ice would be in the form of very small crystals that might remain in the stratosphere long enough to affect the global heat balance.

In an air parcel undergoing rapid vertical lifting (say, greater than $30 \mathrm{~m} \mathrm{~s}^{-1}$ ), the condensate does not have time to grow to precipitation sizes; therefore, almost all the water vapor present at the cloud base will be deposited in the stratosphere in the form of small ice particles. Also, in a strong updraft the air may remain supersaturated with respect to water preventing water drops from evaporating and depositing on to ice particles, a process characteristic of the Bergeron-Findeisen mechanism of precipitation initiation. In an air parcel undergoing a slower rate of lifting, some of the condensate may have time to grow big enough to fall out of the parcel; therefore only a fraction of the water vapor present at the cloud base may be deposited at high levels in the form of small ice particles. 


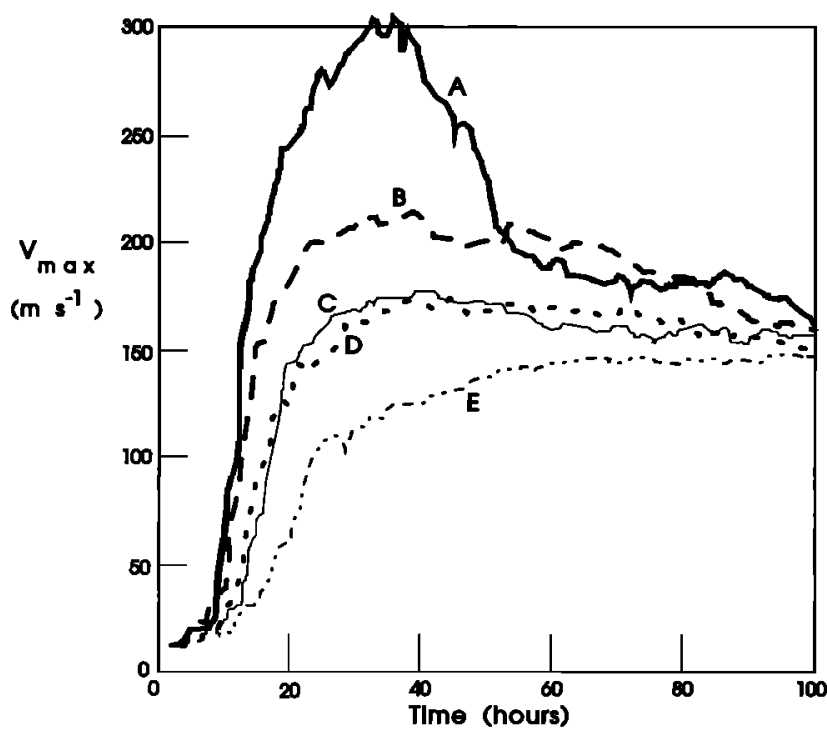

Figure 5. Evolution of the maximum azimuthal velocity in five experiments. Curve $\mathbf{A}$, the main experiment with a maximum temperature of $50^{\circ} \mathrm{C}$ and decay scale of $100 \mathrm{~km}$; curve $\mathrm{B}$, maximum temperature of $47^{\circ} \mathrm{C}$ and decay scale of $100 \mathrm{~km}$; curve $\mathrm{C}$, maximum temperature of $42^{\circ} \mathrm{C}$ and decay scale of 100 $\mathrm{km}$; curve $\mathrm{D}$, maximum temperature of $47^{\circ} \mathrm{C}$ and decay scale of $50 \mathrm{~km}$; and curve $\mathbf{E}$, maximum temperature of $47^{\circ} \mathrm{C}$ and decay scale of $25 \mathrm{~km}$.

What happens to small ice crystals deposited in the middle stratosphere? Let us begin by estimating the average size of the ice particles deposited in the stratosphere. Cloud microphysical calculations show that when an unmixed air parcel is lifted beyond the saturation level, the air becomes supersaturated; the supersaturation reaches a maximum at a short height above, and thereafter it decreases slowly with continued lifting of the parcel (see, for example, Rogers and Yau [1989]). In an unmixed air parcel, in which condensation is the only process operating, the concentration of cloud drops (expressed as number of particles per unit mass of air) is determined at the point of the maximum supersaturation, and thereafter it remains constant with height; furthermore, with continued condensation the size distribution of the cloud drops tends to be monodisperse. In this approximation the particle radius $r$, the mixing ratio $M$, and the particle concentration $n$ are related by

$$
M=\left(4 \pi \rho_{\imath} / 3\right) n r^{3},
$$

where $\rho_{\text {, is }}$ the density of the ice particles. For a rapidly lifted parcel the ice mixing ratio may be taken equal to the saturation water vapor mixing ratio at the cloud base level (considering that there is no fallout and that the saturation mixing ratio is small in the stratosphere); for a slowly lifted parcel the "average" radius may be calculated from (2), provided that $M$ is taken to be the actual mixing ratio of the condensate in the stratosphere and that we have an estimate of the particle concentration.

Twomey (1959) derived the following approximate equation for the concentration $N$ of cloud drops:

$$
N=\alpha 2 /(\beta+2) f_{1} f_{2}(\beta) w 3 \beta / 2(\beta+2),
$$

where $w$ is the vertical velocity of the air. Twomey assumed a power law spectrum of the critical supersaturation of conden-

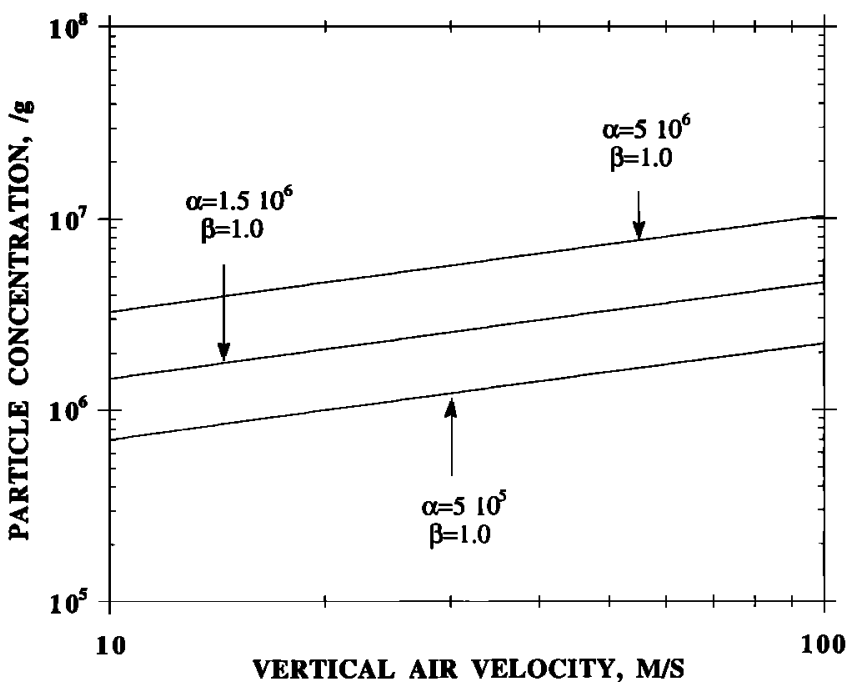

Figure 6. Cloud particle concentration as a function of vertical air velocity for three nucleus spectra.

sation nuclei; that is, he assumed that the concentration of nuclei activated at supersaturation $\sigma$ is $\alpha \sigma^{\beta}$, where $\alpha$ and $\beta$ are constants that depend on the characteristics of the cloud condensation nuclei. In (3), $f_{1}$ depends upon the cloud base temperature and pressure while $f_{2}$ is a function of $\beta$. The power law is in fair agreement with observations; $\alpha$ and $\beta$ are found to range from about 20 to $3000 \mathrm{~cm}^{-3}$ and 0.4 to 1.2 , respectively, when $\sigma$ is expressed in percent [Braham, 1976]. Maritime aerosols have smaller values of $\alpha$, while continental and polluted aerosols have higher values. There is also a tendency for $\boldsymbol{\beta}$ to be larger for maritime aerosols. For our purposes the predominant effect is the dependence of $N$ on $\alpha$ and the vertical air velocity $w$. Figure 6 shows the cloud particle concentration as a function of the vertical air velocity for three nuclei spectra. We see that the cloud particle concentration varies from about $10^{6} \mathrm{~g}^{-1}$ to $10^{7} \mathrm{~g}^{-1}$. Once the particles are deposited in the stratosphere they can settle slowly, and if the ambient air is unsaturated with respect to the particles they can evaporate while settling.

We now consider the settling time of the particles. First let us consider the settling time in the absence of vertical air motion and particle evaporation. Except for the very largest particles considered above, the particle terminal fall speed $v$ is given by the Stokes formula:

$$
v=\left(2 \rho_{\iota} g / 9 \eta\right) r^{2},
$$

where $g$ is the gravitational acceleration and $\eta$ the dynamic viscosity of air. Calculations show that particles of radii 3, 10, 30, and $60 \mu \mathrm{m}$ will take about 2216 hours (92 days), 199.4 hours ( 8.3 days), 22.16 hours ( 0.92 days), and 5.55 hours $(0.23$ days), respectively, to settle through $10 \mathrm{~km}$. Thus these particles can reside in the stratosphere for time periods ranging from a day to months if sedimentation is the only process operating to remove the particles.

However, as updraft air comes to rest in the stratosphere and mixes with the ambient air, or as the particles settle out of the parcel into the ambient air, they will tend to evaporate if the ambient air is unsaturated. The rate of change of particle mass is $m$, given by 


$$
\frac{d m}{d t}=\frac{4 \pi r D \rho_{s}}{1+\gamma} S, \quad \gamma \equiv \frac{L D}{k} \rho_{s}^{\prime},
$$

where $D$ is the diffusivity of water vapor in air, $\rho_{s}$ is the saturation vapor density in the ambient air (with respect to ice), $L$ is the latent heat of sublimation, $k$ is the thermal conductivity of air, $\rho_{s}^{\prime}$ is the derivative of $\rho_{s}$ with respect to the temperature, and $S$ is the supersaturation with respect to the particle. (Equation (5) involves certain approximations; it is accurate only for small magnitudes of $S$. However, for our purposes it should be adequate even for considerable subsaturations.) For an approximate calculation we assume constant $S$ and constant $D, \rho_{s}$, and $\gamma$; for the isothermal stratosphere the latter is a good approximation except for $D$, which is inversely proportional to the pressure. With these approximations, (5) gives

$$
r^{2}=r_{0}^{2}+\frac{2 D \rho_{s}}{(1+\gamma) \rho_{t}} S t
$$

where $r_{0}$ is the initial radius. For subsaturated conditions the time $\tau_{e}$ to completely evaporate a particle of radius $r_{0}$ is given by

$$
\tau_{e}=\frac{(1+\gamma) \rho_{t}}{2 D \rho_{s}} \frac{r_{0}^{2}}{1-\mathscr{H}_{t}}
$$

where $\mathscr{H}_{l}$ is the ambient relative humidity with respect to ice. A particle of initial radius less than $60 \mu \mathrm{m}$ will evaporate completely in less than 5.6 hours in an ambient relative humidity of $99 \%$, assuming $T=220 \mathrm{~K}$ and $p=25$ mbar. Since the diffusion coefficient is inversely proportional to the pressure, the time to complete evaporation would be doubled (quadrupled) if we had used the value of $D$ for $p=50$ mbar (100 mbar), keeping $T=220 \mathrm{~K}$. The time to complete evaporation is also inversely proportional to $\rho_{s}$; therefore using a temperature lower than $220 \mathrm{~K}$ will tend to increase the evaporation time, not only because of a decrease in $\rho_{s}$, but also because with pressure fixed, $D$ decreases with decreasing temperature.

If we consider sedimentation and evaporation simultaneously, (4) and (6) can be used to derive the following expression for the distance of fall in which the particle evaporates completely (assuming no vertical air motion):

$$
h_{f}=\frac{\rho_{t} g}{18 \eta} \frac{(1+\gamma) \rho_{t}}{D \rho_{s}} \frac{r_{0}^{4}}{1-\mathscr{H}_{t}} .
$$

Figure 7 shows the evaporation distance as a function of the initial radius for three selected relative humidities. (Again, we have used $T=220 \mathrm{~K}, p=25 \mathrm{mbar}$; the above remarks on the effects of changing pressure and temperature on $\tau_{e}$ apply to $h_{f}$ as well.) It should be noted that the Stokes fall velocity law on which (8) is based breaks down for particle radii exceeding about $50 \mu \mathrm{m}$. We see that a particle of initial radius $50 \mu \mathrm{m}$ can evaporate completely in a fall through $2 \mathrm{~km}$ in a relative humidity of $99 \%$. Thus we see that most of the condensate lofted high into the stratosphere is likely to evaporate in the stratosphere and increase its vapor content, until and unless the stratosphere becomes saturated.

Assuming that $1 \mathrm{~g} \mathrm{~kg}^{-1}$ of water substance exists in the ring of convection as it enters the middle stratosphere and that the updraft characteristics are those described above, then the flux of water into the middle stratosphere is about $10^{7} \mathrm{~kg} \mathrm{~s}^{-1}$. This is sufficient to completely saturate the layer between 100 and 50 mbar in about 20 days. Were this to happen, the lower and

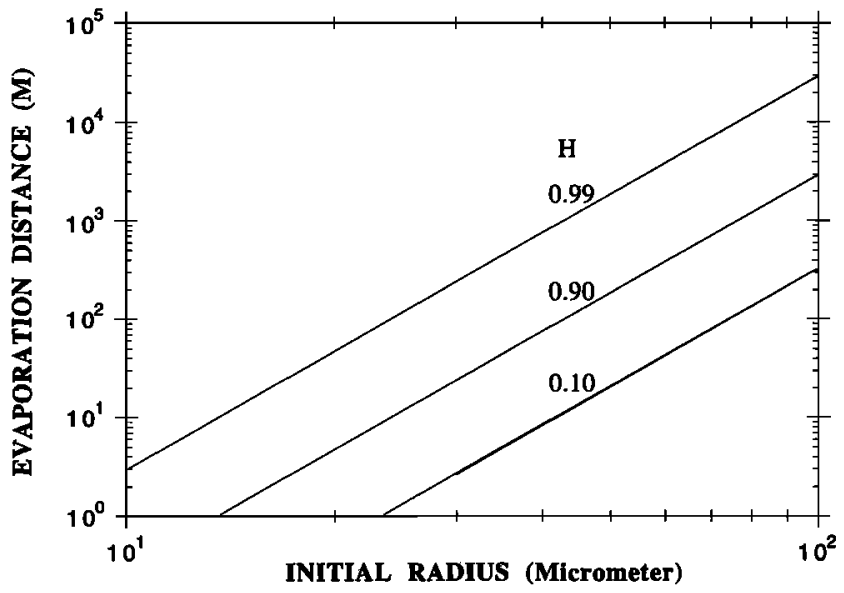

Figure 7. Fall distance to complete evaporation as a function of initial radius for three relative humidities.

middle stratosphere might become cloudy, with profound consequences for global climate. Even if the condensed water were removed from the stratosphere on fairly short timescales, the saturated air would remain.

The injection of large amounts of water into the stratosphere may have significant consequences for the chemistry of that region. Water vapor is the source of the free radicals $\mathrm{OH}$ and $\mathrm{HO}_{2}$, which contribute to stratospheric ozone depletion through the following catalytic cycle:

$$
\begin{gathered}
\mathrm{OH}+\mathrm{O}_{3} \rightarrow \mathrm{HO}_{2}+\mathrm{O}_{2} \\
\mathrm{HO}_{2}+\mathrm{O}_{3} \rightarrow \mathrm{OH}+2 \mathrm{O}_{2} \\
\text { Net: } 2 \mathrm{O}_{3} \rightarrow 3 \mathrm{O}_{2}
\end{gathered}
$$

This is the reaction couplet that contributes most to ozone destruction in the lower stratosphere; it is followed in importance by catalytic cycles involving nitrogen oxides and halogen free radicals. The $\mathrm{OH}$ radical plays also another important role: It activates chlorine (by converting the relatively stable $\mathrm{HCl}$ species to $\mathrm{Cl}$ atoms) and deactivates nitrogen (by converting nitrogen dioxide to nitric acid, a more stable species), the net effect being enhanced ozone depletion by chlorine free radicals.

An important separate effect of water on stratospheric chemistry could result from the formation of clouds: Chemical reactions on cloud droplets activate chlorine and deactivate nitrogen oxides, in a manner analogous to that described above for the $\mathrm{OH}$ radical. Such a mechanism explains the formation of the Antarctic ozone hole. Furthermore, over Antarctica, precipitation of the cloud particles leads to the irreversible removal of nitrogen oxides, because they scavenge nitric acid. This removal sets the stage for efficient ozone destruction by halogen free radicals, a process that slows down in the presence of nitrogen oxides.

Stratospheric clouds are, however, rather scarce and are normally present only over the poles in the winter or spring months, which is where ozone depletion has taken place in recent years. These observations indicate that the presence of stratospheric clouds at low latitudes could have profound consequences for ozone depletion. The main natural source for chlorine in the stratosphere is methyl chloride, produced in the biosphere; if additional amounts of chlorine were to be in- 
jected, for example, from sea salt, the effects on stratospheric ozone could be dramatic.

\subsection{Aerosol Injection}

The large vertical velocities and mass flux rates sustained in hypercanes are capable of injecting large amounts of aerosol into the middle stratosphere. Bolide impact might generate large amounts of fine dust by excavation, while undersea volcanism produces ash. The injection of volcanic ash or terrestrial material excavated by bolide impact into the middle stratosphere can be expected to influence climate for several years to a decade, given the observed presence of volcanic material in the stratosphere for several years following major historical eruptions. The largest of historical eruptions have led to measurable global cooling [Budyko et al., 1988].

\section{Bolide Impact, Undersea Volcanism, and Hypercane Formation}

The numerical simulations described in section 3 suggest that hypercanes might occur over localized regions of water heated to peak temperatures in excess of about $45^{\circ} \mathrm{C}$ on scales greater than about $50 \mathrm{~km}$. Such regions could only initiate hypercanes if the ambient atmospheric conditions were otherwise favorable for hurricane formation; that is, the atmosphere would have to have nearly moist adiabatic lapse rates through most of the troposphere and could not contain much vertical wind shear. In addition, the mean wind speed would have to be small, particularly in the case of small-scale sea surface temperature anomalies, so that the developing storm would not move rapidly away from the sea surface temperature anomaly.

All of these conditions suggest that hypercanes would be extremely rare, occurring only when the ocean surface is heated to an extraordinary degree somewhere in the tropics, and then only under ideal conditions. Solar heating is not capable of raising sea surface temperatures to the necessary degree, and thus other heat sources must be considered.

In the hypothetical impact scenario envisioned by Emiliani et al. [1981], a 14-km-diameter body of mass $2.5 \times 10^{15} \mathrm{~kg}$ hits the Earth with a speed $2 \times 10^{4} \mathrm{~m} \mathrm{~s}^{-1}$. Only a tiny fraction $(0.1 \%)$ of the initial kinetic energy, $5 \times 10^{23} \mathrm{~J}$, is lost as the bolide moves through the atmosphere; the principal transfer to the atmosphere occurs via the plume thrown up by the impact, estimated at $25 \%$ of initial kinetic energy. The remaining $75 \%$ is available to excavate the impact crater in the ocean and solid earth and to heat the rock and seawater. It is thought that such an impact would create a crater roughly $100 \mathrm{~km}$ in diameter and $35 \mathrm{~km}$ deep at its center, exposing underlying mantle. As the ocean rushes back over the rim to fill in the crater over a period of hours, it is heated mainly by contact with hot or molten rock. This direct heating depends crucially on the degree of heat exchange between the hot debris, upwelling magma, and seawater.

Subtracting the energy going into the plume, the total energy available from the collision is $4 \times 10^{23} \mathrm{~J}$, including that liberated from the warm crust and mantle [Emiliani et al., 1981]. Using this energy to heat seawater in a layer $4 \mathrm{~km}$ thick $(4 \times$ $10^{13} \mathrm{~m}^{3}$ ), the ocean temperature in the crater $100 \mathrm{~km}$ in diameter could rise by $1000^{\circ} \mathrm{C}$, that is, create a column of boiling water with sea surface temperature near $100^{\circ} \mathrm{C}$ (higher at depth owing to pressure).

Emiliani et al. [1981] noted that direct heating of the ocean by lava outflow is attenuated because of the tendency for mol- ten rock to be insulated from seawater by a thin solid crust, effectively reducing the heat transfer rate. However, they also describe the probable intimate mixing of hot debris with seawater as the ocean rushes back into the crater. Furthermore, although they discount a large-scale, ocean-wide heating, because of the tendency for rotational constraints to confine the hot column locally, it is precisely this confinement which encourages the high sea surface temperature (SST) needed for hypercane formation.

To investigate the nature of this rotational confinement with strong heating, we consider a less dramatic event: a volcanic eruption within the deep Red Sea hot brine pools. This provides us with a real, existing natural laboratory for testing ideas about the mixing and confinement of superheated seawater.

The brine pools are reservoirs of hot, salty fluid which accumulate around geothermal sources at the floor of the Red Sea. Their extreme saltiness turns them into huge heat storage devices in which temperature can build up, since their density remains high enough for them to rest on the bottom. Typically, the observed temperature is above $60^{\circ} \mathrm{C}$ and salinity is above 250 practical salinity units (psu) [Ross, 1983], giving a density of about $1200 \mathrm{~kg} \mathrm{~m}^{-3}$. The overlying deep water, at $22^{\circ} \mathrm{C}$ and $40.5 \mathrm{psu}$, has a density close to $1030 \mathrm{~kg} \mathrm{~m}^{-3}$. To make the dense brine buoyant, its temperature must be raised above $300^{\circ} \mathrm{C}$ by volcanic activity. An estimate of brine volume in the Atlantis Deep Basin, Chain Basin, Discovery Basin, and nearby smaller basins is $7 \mathrm{~km}^{3}$ [Blanc et al., 1990; J.-L. Charlou, personal communication, 1994]. The possibility for a substantial volume of water to become superheated before becoming buoyant suggests that even after dilution with the surrounding seawater in the rising plume, the final temperature anomaly may still be very high.

To explore the dynamical possibility that this hot brine reaches the surface in some diluted form, we adapted a threedimensional nonhydrostatic numerical model. This model has been described and applied to convection in the Mediterranean Sea by Jones and Marshall [1993] and to hydrothermal plumes by Speer and Marshall [1995]. A nonhydrostatic model is necessary in our case to resolve explicitly the processes which produce dilution and mixing with strong vertical motion and to avoid making assumptions about entrainment. Earth's rotation constrains the lateral expansion of the plume in the sea and is expected to influence the long-term evolution of the volcanic plume.

The domain is a periodic box $32 \mathrm{~km} \times 32 \mathrm{~km} \times 2000 \mathrm{~m}$, with heating distributed over a disk $8 \mathrm{~km}$ in diameter at the bottom, representing a volcanic eruption in the basins. The grid is 132 $\times 132 \times 20$, for a grid size of $250 \mathrm{~m}$ in the horizontal and 100 $\mathrm{m}$ in the vertical. Initially, there is a layer of hot brine one grid box thick on the bottom with neutral buoyancy; that is, it is assumed that heating has already raised the temperature of the brine to the point of convective overturning. The horizontal mixing coefficients are all set to $5 \mathrm{~m}^{2} \mathrm{~s}^{-1}$, and the vertical coefficients are $0.2 \mathrm{~m}^{2} \mathrm{~s}^{-1}$. These small values ensure that mixing occurs mainly by advection.

To estimate the forcing strength, an eruption documented on the Juan de Fuca Ridge cleft segment is used as a reference [Fox et al., 1992]. In this case, $0.05 \mathrm{~km}^{3}$ of magma erupted covering approximately $2.1 \mathrm{~km}^{2}$ of seafloor. A total heat transfer of about $1.5 \times 10^{11} \mathrm{~J} \mathrm{~m}^{-2}$ occurred as this lava flow cooled to ambient temperature. For a thickness of order $d=10 \mathrm{~m}$, the timescale for cooling is of order $d^{2} / 4 \kappa$, giving $10^{7} \mathrm{~s}$ for a conductivity $\kappa=10^{-6} \mathrm{~m}^{2} \mathrm{~s}^{-1}$. Then the heat flux into the 


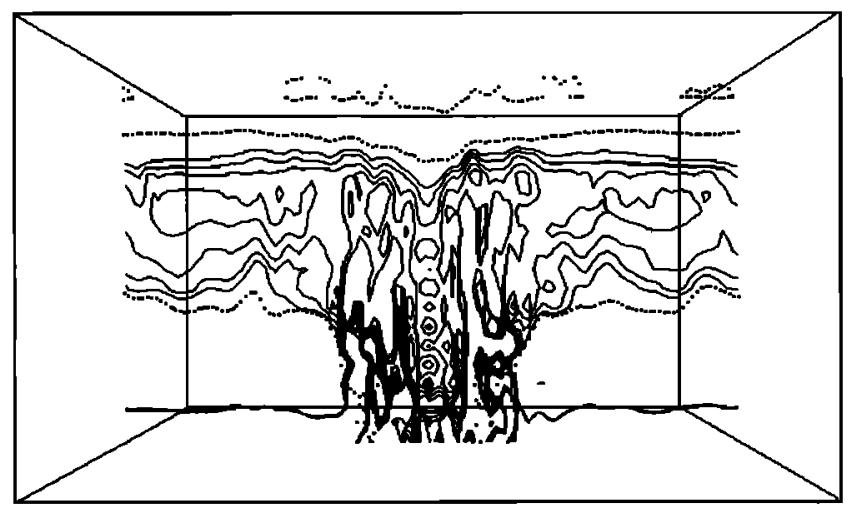

Figure 8. Simulation of a Red Sea hot brine plume created by a volcanic heat source on the bottom. The domain is $32 \mathrm{~km}$ on a side and $2 \mathrm{~km}$ deep. Simulation shows contours of salinity (solid lines) at an interval of $2.5 \mathrm{psu}$ from $60-70 \mathrm{psu}$ and density (dashed lines) at an interval equivalent to $1^{\circ} \mathrm{C}$. The corresponding maximum temperature anomaly is roughly $40^{\circ} \mathrm{C}$. The brine rises off the bottom as a plume up to the base of the thermocline, where it spreads laterally. After a period of about 1 day the plume is circulating anticyclonically (clockwise). After a few days the plume reaches the edge of the domain.

ocean is of order $10^{4} \mathrm{~W} \mathrm{~m}^{-2}$ on average. To represent a strong eruption, a heat flux value of $H=50 \mathrm{~kW} \mathrm{~m}^{-2}$ was chosen. The equivalent buoyancy flux is $B=g \alpha H / \rho c_{p}=4.5 \times 10^{-5} \mathrm{~m}^{2}$ $\mathrm{s}^{-3}$, and the total buoyancy input integrated over the source area is $F=2700 \mathrm{~m}^{4} \mathrm{~s}^{-3}$.

The stratification of the Red Sea is relatively weak below 500 $\mathrm{m}$ depth, where the buoyancy frequency $N_{\text {deep }}=7 \times 10^{-4} \mathrm{~s}^{-1}$, but there is a strong thermocline in the upper $500 \mathrm{~m}$, where $N_{\text {therm }}=7 \times 10^{-3} \mathrm{~s}^{-1}$. Using a Coriolis parameter appropriate for midbasin (latitude $20^{\circ} \mathrm{N}$ ), $f=5 \times 10^{-5} \mathrm{~s}^{-1}$, the large ratio $N_{\text {therm }} / f=140$ or $N_{\text {deep }} / f=14$ shows that stratification and not rotation controls the height of penetration. The penetration height is therefore $Z_{N}=3.8\left(F / N^{3}\right)^{1 / 4}$ above a virtual source several kilometers below the real seafloor. For the above forcing and $N_{\text {deep }}, Z_{N}=6400 \mathrm{~m}$, sufficient to reach the base of the thermocline. At the base of the thermocline a point source approximation is no longer appropriate for scaling purposes.

A numerical simulation of an eruption into the brine pools shows the generation of a hot brine plume at intermediate depths in the Red Sea (Figure 8). Numerical constraints precluded the use of realistic brine temperature and salinity, so salinity was used as a proxy tracer for mixing; the final temperature of the equilibrium plume as determined by salinities of $40-100 \mathrm{psu}$ is roughly in the range $25-50^{\circ} \mathrm{C}$. Over the rising and spreading period, most of the heat in the plume comes from the brine pools and not the source. This situation is similar to that of the megaplumes which have been observed above the midocean ridge in the Pacific Ocean [Baker et al, 1989]. After one day of integration the radial motion of water toward the source at depth and away from the source at the spreading level has generated a vortex, anticyclonic at intermediate depths and cyclonic in deep water, owing to rotation. The vortex holds together a lens of warm brine above the source. Despite this confinement and recirculation of the plume, it still does not reach the surface after several days of integration. Runs with similar stratification and source geometry showed similar behavior.

The buoyancy flux from the source, though large compared to inactive ridge crest fluxes, is simply too weak to drive the plume across the thermocline. (Note that the largest volcanic plumes in the atmosphere easily penetrate the stratosphere [Sparks, 1986]. The analogous thermocline, the tropopause, is only roughly double the stratification in the troposphere and does not appear to form much of a barrier.) A penetration scale based on buoyancy flux is $\left(B / N_{\text {therm }}^{3}\right)^{1 / 2}$, which is only 12 $\mathrm{m}$. A source 1000 times stronger would appear to be strong enough to penetrate the thermocline; the total source strength would then be $3.2 \times 10^{15} \mathrm{~W}$, placing it among the largest eruptions observed on land [Settle, 1978; Wilson et al., 1978]. In the present model configuration, such large forcing is impossible to simulate as it leads to numerical instabilities. We conclude that the dynamical constraint of rotation is important to form a strong anomaly and that these anomalies can attain the surface in the case of very powerful, but not unprecedented, volcanic sources.

We note parenthetically that isopycnals at intermediate depths outcrop at the northern end of the Red Sea, so if the subsurface hot plume is carried north in the general circulation, it is conceivable that it may eventually reach the surface following isopycnals. It is interesting that even modest eruptions are capable of forming a hot brine plume at intermediate depths.

On the other hand, a straightforward way for volcanism to heat the surface layer of the ocean is for it to occur in shallow water, as in the first stages of island formation. To raise the temperature of an ocean surface layer $50 \mathrm{~m}$ thick and $50 \mathrm{~km}$ on a side $\left(125 \mathrm{~km}^{3}\right)$ from $25^{\circ} \mathrm{C}$ to $50^{\circ} \mathrm{C}$ requires $1.3 \times 10^{19} \mathrm{~J}$. To supply this heat over an eruption timescale of 10 hours [Wilson et al., 1978] implies a thermal source of $3.4 \times 10^{14} \mathrm{~W}$. At $100 \%$ efficiency of heat transfer from magma to seawater, the corresponding magma flow rate is about $6 \times 10^{4} \mathrm{~m}^{3} \mathrm{~s}^{-1}$ (assuming $\rho c_{p}=3 \times 10^{6} \mathrm{~J} \mathrm{~m}^{3}{ }^{\circ} \mathrm{C}$, an initial temperature of $1000^{\circ} \mathrm{C}$, and an equal heating contribution from latent heat of freezing). Lower efficiencies imply higher flow rates, but even higher flow rates are not unknown in existing volcanoes [Settle, 1978].

The nature of the eruption will determine whether or not the heat transfer efficiency is high. Explosive eruptions are more effective at mixing pyroclast with surrounding fluid; slower eruptions have to cover a large area with thin layers of lava in order to generate fluxes of the order $10^{4} \mathrm{~W} \mathrm{~m}^{-2}$, capable of adequately heating the overlying water column tens of meters deep in a period of days. Thus high efficiency is not necessary for a strong anomaly, but the quicker the anomaly is formed the less chance that it is dissipated into the background before a storm develops. The above estimates show only the plausibility of a volcanically produced SST anomaly adequate to generate a hypercane. The actual probability of satisfying the various conditions is difficult to determine.

Finally, it should be emphasized that even the large air-sea heat fluxes induced by hypercane generation are nevertheless far too small to erase the sea-surface temperature anomaly in a short time. The maximum surface fluxes produced by the numerical simulation would cool a $100-\mathrm{m}$ column of seawater at the rate of no more than $1^{\circ} \mathrm{C}$ per day. The average fluxes over the warm pool would cool the same column by about $0.1^{\circ} \mathrm{C}$ per day. Thus it would take several weeks to reduce the seawater temperature to below the hypercane threshold, even 
if there were no continuing heat fluxes into the ocean from the volcanic or bolide impact-induced source.

\section{Summary}

The two principal scenarios for rapid global extinctions, bolide impact and extensive volcanism, both rely to some degree on stratospheric effects to produce worldwide ecosystem stress. We regard with skepticism claims that the mechanical effects of bolide impact would directly lead to large-scale contamination of the stratosphere or that the heating effects of such an impact or the wildfires that result from it could lead to significant injection of mass into the stratosphere. Similarly, the weak dependence of the maximum penetration altitude of turbulent convective plumes on the surface heat flux casts doubt on whether even unusually large volcanic events could disrupt climate much beyond what has been observed in historical eruptions. We propose instead that some instances of undersea volcanism or oceanic bolide impact could lead to the formation of hypercanes capable of injecting large amounts of material into the middle stratosphere. The contamination of the normally dry stratosphere with water may have large transient effects on climate through the direct absorption of infrared radiation by water vapor and through the radiative effects of large-scale stratospheric cloudiness, and may lead to serious depletion of ozone and thus to increased ultraviolet radiation at the surface. The injection of large quantities of aerosol into the middle stratosphere may also have strong effects on climate.

The necessary conditions for the formation of normal hurricanes lead us to conclude that hypercane formation would be limited to the tropics, even in the case of very large anomalies of sea surface temperature. If our hypothesis has any merit, it suggests that global extinctions will be somewhat more frequent in the case of oceanic bolide impact or undersea volcanism in the tropics. This prediction may provide some means of empirically testing the theory.

\section{References}

Alt, D., J. M. Sears, and D. W. Hyndman, Terrestrial maria: The origins of large basalt plateaus, hotspot tracks and spreading ridges, J. Geol., 96, 647-662, 1988.

Alvarez, L. W., W. Alvarez, F. Asaro, and H. V. Michel, Extraterrestrial cause for the Cretaceous-Tertiary extinction, Science, 208, 1095-1108, 1980.

Baker, E. T., J. W. Lavelle, R. A. Feely, G. J. Massoth, and S. L. Walker, Episodic venting of hydrothermal waters from the Juan de Fuca Ridge, J. Geophys. Res., 94, 9237-9250, 1989.

Blanc, G., J. Boulègue, and J.-L. Charlou, Profils d'hydrocarbure légers dans l'eau de mer, les saumures et les eaux interstitielles de la fosse Atlantis II (mer Rouge), Oceanol. Acta., 13, 187-197, 1990.

Braham, R. R., CCN spectra in C-k space, J. Atmos. Sci., 33, 343-345, 1976.

Budyko, M. I., G. S. Golitsyn, and Y. A. Izrael, Global Clımate Catastrophes, 99 pp., Springer-Verlag, New York, 1988.

D'Hondt, S., and G. Keller, Some patterns of planktonic foraminiferal assemblage turnover at the Cretaceous/Tertiary boundary, Mar. Micropaleontol., 17, 77, 1991.

Emanuel, K. A., An air-sea interaction theory for tropical cyclones, I, J. Atmos. Sci., 42, 1062-1071, 1986.

Emanuel, K. A., The maximum intensity of hurricanes, J. Atmos. Sci., $45,1143-1155,1988$.

Emanuel, K. A., The finite-amplitude nature of tropical cyclogenesis, $J$. Atmos. Sci., 46, 3431-3456, 1989.
Emiliani, C., E. B. Kraus, and E. M. Shoemaker, Sudden death at the end of the Mesozoic, Earth Planet. Sci. Lett., 55, 317-334, 1981.

Fox, C. G., W. W. Chadwick, and R. W. Embley, Detection of changes in ridge-crest morphology using repeated multibeam sonar surveys, J. Geophys. Res., 97, 11,149-11,162, 1992.

Hsü, K. J., Terrestrial catastrophe caused by cometary impact at the end of Cretaceous, Nature, 285, 201-203, 1980.

Jones, $H$., and J. Marshall, Convection with rotation in a neutral ocean: A study of open-ocean deep convection, J. Phys. Oceanogr., 23, 1009-1039, 1993.

Luck, J. M., and K. K. Turekian, Osmium-187/osmium-186 in manganese modules and the Cretaceous/Tertiary boundary, Science, 222, 613-615, 1983.

Morton, B. R., G. I. Taylor, and J. S. Turner, Turbulent gravitational convection from maintained and instantaneous sources, Proc. $R$. Soc. London A, 234, 1-23, 1956.

O'Keefe, J. D., and T. J. Ahrens, Impact production of $\mathrm{CO}_{2}$ by the Cretaceous/Tertiary extinction bolide and the resulting heating of the earth, Nature, 338, 247, 1989.

Officer, C. B., and C. L. Drake, The Cretaceous-Tertiary transition, Science, 219, 1383-1390, 1983.

Prinn, R. G., and B. Fegley, Bolide impacts, acid rain and biospheric traumas at the Cretaceous/Tertiary boundary, Earth Planet. Sci. Lett., 83, 1-15, 1987.

Reitmeijer, F. J. M., Volcanic dust in the stratosphere between 34 and $36 \mathrm{~km}$ altitude during May, 1985, J. Volcanol. Geotherm. Res., 55, 69-83, 1993.

Rogers, R. R., and M. K. Yau, A Short Course in Cloud Physics, 293 pp., Pergamon, Tarrytown, N. Y., 1989.

Ross, D. A., The Red Sea, in Estuaries and Enclosed Seas, edited by B. H. Ketchum, pp. 293-307, Elsevier, New York, 1983.

Rotunno, R., and K. A. Emanuel, An air-sea interaction theory for tropical cyclones, II, J. Atmos. Sci., 44, 542-561, 1987.

Settle, M., Volcanic eruption clouds and thermal power output of explosive eruptions, J. Volcanol. Geotherm. Res., 3, 309-324, 1978.

Sparks, R. S. J., The dimensions and dynamics of volcanic eruption columns, Bull. Volcanol., 48, 3-15, 1986.

Speer, K., and J. Marshall, Convection from seafloor hot springs, $J$. Mar. Res., in press, 1995.

Thierstein, H. R., Late Cretaceous nannoplankton and the change at the Cretaceous-Tertiary boundary, in The Deep Sea Drilling Project: $A$ Decade of Progress, edited by J. Wamme, E. L. Winteres, and R. G. Douglas, Spec. Publ. Soc. Econ. Paleontol. Mineral., 32, 355-394, 1981 .

Twomey, S., The nuclei of natural cloud formation: The supersaturation in natural clouds and the variation of cloud droplet concentration, Geofis. Pura Appl., 43, 243-249, 1959.

Ward, P., Maastrichtian ammonite and inoceramid ranges from Bay of Biscay Cretaceous/Tertiary boundary sections, in Paleontology and Evolution: Extinction Events, edited by M. Lamolda, E. Kauffman, and O. Walliser, pp. 119-126, Soc. Espan. de Paleontol., Madrid, 1988.

Wilson, L., R. S. J. Sparks, T. C. Huang, and N. D. Watkins, The control of eruption column heights by eruption energetics and dynamics, J. Geophys. Res., 83, 1829-1836, 1978.

Wolbach, W. S., R. S. Lewis, and E. Anders, Cretaceous extinctions: Evidence for wildfires and search for meteoric material, Science, 230, 167-170, 1985.

Zahnle, K., and D. Grinspoon, Comet dust as a source of amino acids at the Cretaceous/Tertiary boundary, Nature, 348, 157, 1990.

K. A. Emanuel and M. Molina, Center for Meteorology and Physical Oceanography, Massachusetts Institute of Technology, Cambridge, MA 02139.

R. Rotunno, P.O. Box 3000, National Center for Atmospheric Research, Boulder, CO 80307.

K. Speer, Institut Français de Recherche pour l'Exploitation de la Mer, B.P. 70, 29280 Plouzané, France.

R. Srivastara, Department of Geophysical Sciences, The University of Chicago, Chicago, IL 60637.

(Received September 8, 1994; revised March 8, 1995; accepted March 22, 1995.) 